\title{
The number-theoretic content of the Jacobi triple product identity
}

\author{
Herbert S. Wilf \\ Department of Mathematics, University of Pennsylvania \\ Philadelphia, PA 19104-6395
}

\begin{abstract}
We prove the Jacobi Triple Product Identity by exhibiting an elementary number-theoretic proposition that is equivalent to it, and then proving that the proposition is true.
\end{abstract}

Dedicated to George Andrews on his sixtieth birthday:

"Unsolved problems tremble with fear as he approaches." 
The triple product identity of Jacobi asserts that

$$
\sum_{n=-\infty}^{\infty} z^{n} q^{n^{2}}=\prod_{n \geq 0}\left(1-q^{2 n+2}\right)\left(1+z q^{2 n+1}\right)\left(1+z^{-1} q^{2 n+1}\right) .
$$

Many proofs of the JTPI are known. There is a proof in Andrews's memoir [2], along with an extensive list of references, and another one by George Andrews in [1], which uses two identities of Euler. A proof of E.M. Wright [5] is combinatorial, and involves a direct bijection of bipartite partitions. Proofs due to Cheema [3] and to Sudler [4] are variations of the one of Wright cited above. Here we give one which proceeds by finding an unusual proposition in elementary number theory to which the Jacobi identity is equivalent, and proving it.

To discover the number-theoretic identity that is equivalent to (1), do the following:

1. operate on the logarithm of both sides with $q \partial / \partial q$,

2. multiply by the left side of (1) to clear of fractions, and

3. equate the coefficients of $z^{a} q^{b}$ on both sides.

The identity that results can be written as

$$
\begin{gathered}
\sum_{d \in S(n, 1-2 a)}(-1)^{d-1}\left\{\frac{n}{d}-d+2 a\right\}+\sum_{d \in S(n, 1+2 a)}(-1)^{d-1}\left\{\frac{n}{d}-d-2 a\right\} \\
= \begin{cases}2 \sigma(n / 2), & \text { if } n \neq 0 ; \\
a^{2}, & \text { if } n=0,\end{cases}
\end{gathered}
$$

where we have written $n=b-a^{2}$, and

$$
S(n, x)=\left\{d: d \backslash n \text { and } \frac{n}{d}-d \text { is odd and } \frac{n}{d}-d \geq x\right\},
$$

and $\sigma(N)$ is the sum of the divisors of $N$, if $N$ is a positive integer, and is 0 otherwise.

Note that $b$ does not appear explicitly in (2). In fact, we claim that (2) is true for all integers $n$ and all real $a$. We prove it for $n>0$ and all real $a$, the case $n<0$ being similar, and $n=0$ being easy.

Indeed, if $n$ is odd then the left side of (2) vanishes and the sums on the right side are empty, so the assertion is trivially true. Hence we can suppose that $n$ is even. 
To prove that (2) holds for all integers $a$ we will now show that it holds for all sufficiently large $a$ and then that its truth for $a$ implies the same for $a-1$.

First, for $n$ fixed, $\exists a_{0}$ such that $\forall a>a_{0}$ the second sum on the right of (2) is empty, and for such $a$ we must show that

$$
2 \sigma(n / 2)=\sum_{d \in S(n,-\infty)}(-1)^{d-1}\left\{\frac{n}{d}-d\right\}+2 a \sum_{d \in S(n,-\infty)}(-1)^{d-1} .
$$

Let $n=2^{m} n^{\prime}, n^{\prime}$ odd, $m \geq 1$. Then the right side of (3) is

$$
\begin{aligned}
& \sum_{d \backslash n^{\prime}}\left\{2^{m} \frac{n^{\prime}}{d}-d\right\}-\sum_{d^{\prime} \backslash n^{\prime}}\left\{\frac{n^{\prime}}{d^{\prime}}-2^{m} d^{\prime}\right\}+(2 a) \sum_{d \backslash n^{\prime}} 1+(2 a) \sum_{d^{\prime} \backslash n^{\prime}}(-1) \\
& =2^{m} \sigma\left(n^{\prime}\right)-\sigma\left(n^{\prime}\right)-\sigma\left(n^{\prime}\right)+2^{m} \sigma\left(n^{\prime}\right)=2\left(2^{m}-1\right) \sigma\left(n^{\prime}\right)=2 \sigma(n / 2),
\end{aligned}
$$

so (2) is proved for all large enough $a$. Finally, if $f(a)$ denotes the right side of $(2)$ then we claim that $f$ is constant. Indeed, $f(a+1)-f(a)$ is equal to

$$
\begin{aligned}
& \begin{cases}(-1)^{d}(2 a+1), & \text { if } \exists d \backslash n: \frac{n}{d}-d=2 a+1 ; \\
0, & \text { otherwise. }\end{cases} \\
& +(2 a+2) \sum_{d \in S(n,-2 a-1)}(-1)^{d-1}-(2 a) \sum_{d \in S(n, 1-2 a)}(-1)^{d-1} \\
& - \begin{cases}(-1)^{d}(2 a+1) & \text { if } \exists d \backslash n: \frac{n}{d}-d=2 a+1 ; \\
0, & \text { otherwise. }\end{cases} \\
& -(2 a+2) \sum_{d \in S(n, 2 a+3)}(-1)^{d-1}+(2 a) \sum_{d \in S(n, 2 a+1)}(-1)^{d-1} \\
& =(2 a+2)\left(\sum_{d \in S(n,-2 a-1)}-\sum_{d \in S(n, 2 a+3)}\right)(-1)^{d-1} \\
& +(2 a)\left(\sum_{d \in S(n, 2 a+1)}-\sum_{d \in S(n,-2 a+1)}\right)(-1)^{d-1} \\
& =2 a\left(\sum_{d \in S(n,-2 a-1)}-\sum_{d \in S(n,-2 a+1)}+\sum_{d \in S(n, 2 a+1)}-\sum_{d \in S(n,-2 a+1)}\right)(-1)^{d-1} \\
& +2\left(\sum_{d \in S(n,-2 a-1)}-\sum_{d \in S(n, 2 a+3)}\right)(-1)^{d-1} .
\end{aligned}
$$

The terms in the four sums inside the first large parenthesis cancel immediately except for two terms, and those are seen at once to cancel. The terms in the second large parenthesis cancel in pairs, and the proof is complete.

My thanks go to Robin Chapman for several helpful comments. 


\section{References}

[1] George E. Andrews, A Simple Proof of Jacobi's Triple Product Identity, Proceedings of the American Mathematical Society, Vol. 16, No. 2. (Apr., 1965), pp. 333-334.

[2] George E. Andrews, Generalized Frobenius partitions, Mem. Amer. Math. Soc. 49 (1984), no. 301, iv+44 pp.

[3] M. S. Cheema, Vector Partitions and Combinatorial Identities, Math. Comp. 18 (1964), 414-420.

[4] C. Sudler, Two enumerative proofs of an identity of Jacobi, Proc. Edinburgh Math. Soc. 15 (1966), 67-71.

[5] E. M. Wright, An enumerative proof of an identity of Jacobi, J. London Math. Soc. 40 (1965), 55-57. 